pathology and post mortem databases for the five hospitals in the network. A full review of the clinical records was performed.

Results 81 patients were identified. Average age was 72 and $94 \%$ were male. $82.7 \%$ of patients had a performance status of 0 or 1 at presentation but $21 \%$ had a significant co-morbidity, most commonly cardiac in nature. $77.8 \%$ of patients had definite or probable asbestos exposure. At presentation symptoms had been present for a mean of 3.7 months, the commonest being breathlessness and chest pain. A definitive diagnosis was made in $84 \%$ of patients, either on histology $(75 \%)$ or cytology $(9 \%)$ and the epithelioid subtype was most common $(40.7 \%$ of cases). VATS was the main diagnostic modality (61.7\%). Despite $61.7 \%$ of patients technically being fit for chemotherapy, only $29.6 \%$ received it. Mean survival was 269 days (8.8 months). Survival was longer in those with a better performance status at presentation ( $\mathrm{PS} 0=344$ days, $\mathrm{PS} 1=270 \mathrm{~d}, \quad \mathrm{PS} 2=125 \mathrm{~d}$, PS3 $=89 d$ ), and in those who received chemotherapy (448d vs 269d). Conclusion MM remains a common problem in the South West of the UK. Despite recent treatment advances the prognosis remains poor, with our survival data being similar to that described in earlier population based studies and in chemotherapy treatment trials. Only a small proportion of our patients received chemotherapy and this may reflect the lack of NICE guidance for use of pemetrexed which was only issued in 2008. However, those patients who recieved chemotherapy had a better survival time (14.7 months vs 8.8 month).

\section{P70 TALC SLURRY PLEURODESIS: DOES MIXING TALC WITH 50\% DEXTROSE RATHER THAN NORMAL SALINE INCREASE SUCCESS RATE?}

doi:10.1136/thx.2010.150979.21

A Khanna, C Mackinlay, A Kerry. Great Western Hospital, Swindon, UK

Introduction Symptomatic malignant pleural effusions can be managed effectively with talc pleurodesis. Various studies document the success rate for talc pleurodesis around $80 \%$. In these studies, talc was mixed with normal saline to make the slurry prior to pleurodesis. We audited the success rate for pleurodesis in our hospital using slurry made by mixing talc with normal saline and slurry made by mixing talc with $50 \%$ dextrose to see if changing the 'solvent' made any difference to the success of the procedure. The hypothesis that $50 \%$ dextrose, being 'stickier' than normal saline, will achieve a better success rate was thus tested.

Method In group 1, 28 patients underwent pleurodesis using slurry obtained by mixing talc with normal saline. The findings were presented to BTS in 2008. Subsequently we re-audited our practice with group 2 , in which 18 patients were pleurodesied using slurry obtained by mixing talc with $50 \%$ dextrose.

Results Procedure success was defined as absence of fluid reaccmulation requiring intervention at 3 months post pleurodesis. The success rate for the first group was 34\% while the success rate for the second group was $76 \%$. The second group underwent more rigorous protocol ensuring drain clamping for 1-h post procedure and use of suction in $100 \%$ compared to $80 \%$ and $87.5 \%$ in the first group. However, subgroup analysis of the first group did not reveal a statistically significant difference in success rate for these variables. Use of NSAID analgesia for $28 \%$ patients in group 1 compared to none in group 2 could have influenced the results significantly. Lastly, better patient selection and operator bias might have resulted in better success rate in the second group as recommendations from first audit were implemented in second cycle. Overall best success rate of $76 \%$ was inferior to that reported in previous studies due to more liberal patient selection.

Conclusions Use of $50 \%$ dextrose rather than normal saline to mix talc for talc slurry with strict patient selection and a rigorous pleurodesis protocol as recommended by BTS guidelines can result in better success rate. This is possibly because of the resulting stickier inert slurry. However, research with a randomised control trial is required to assess this question further.

\section{Paediatric lung diseases \begin{tabular}{l}
\hline P71 REDUCED AIRWAY BETA-DEFENSIN 2 LEVELS IN CHILDREN \\
WITH CYSTIC FIBROSIS AND VITAMIN D-DEFICIENCY
\end{tabular}} doi:10.1136/thx.2010.150979.22

${ }^{1} \mathrm{~K}$ Naderi, ${ }^{2} \mathrm{~J}$ Donovan, ${ }^{2} \mathrm{~S}$ Brown, ${ }^{1} \mathrm{~N}$ Leaver, ${ }^{2} \mathrm{H}-\mathrm{L}$ Tan, ${ }^{1} \mathrm{E}$ W F W Alton, ${ }^{1} \mathrm{~A}$ Bush, 'J C Davies. ${ }^{1}$ Imperial College, London, UK; ${ }^{2}$ Royal Brompton \& Harefield NHS Foundation Trust, London, UK

Vitamin D (vD) levels have been reported to correlate with (a) lung function in healthy populations and (b) disease severity in pulmonary $\mathrm{TB}, \mathrm{COPD}$ and asthma. The proposed mechanism, supported by in vitro studies, relates to $\mathrm{vD}$ response elements in the promoter regions of genes encoding molecules involved in innate immunity such as defensins and cathelicidin (LL-37). As patients with CF are at risk of fat and fat-soluble vitamin malabsorption, we sought to explore this relationship in a cohort of CF children. Frozen serum and bronchoalveolar (BAL) fluid samples, which had been donated for research at the time of a clinically-indicated bronchoscopy were available from 49 children with CF. Mean age at the time of the procedure was 6.8 years (range 0.03-15.99). 44 (90\%) were biochemically pancreatic insufficient and were prescribed pancreatic enzyme supplementation and fat-soluble mulitvitamins. Serum $25 \mathrm{OH}$ vD3 was measured using HPLC and mass spectrometry. BALF human beta defensin-2 (hbD2) and LL-37 were quantified using ELISA. vD deficiency was defined as $<20 \mathrm{ng} / \mathrm{ml}$ based on internationally-accepted criteria. Deficient and sufficient groups were compared with Mann-Whitney tests and Spearman's correlations were performed. $16(33 \%)$ children were vD-deficient (including two of the five pancreatic sufficient patients); they did not differ in age from the vD sufficient group. BALF hbD2 was significantly lower than in the vD sufficient group (median (range) 185.3 (7.8-615.7) $\mathrm{pg} / \mathrm{ml}$ vs $385.5(7.8-1002) \mathrm{pg} / \mathrm{ml} ; \mathrm{p}<0.05)$. In contrast, no relationship was observed between serum vD and BAL LL-37. As this molecule is known to be highly sensitive to proteolysis, we considered the possibility that degradation could be masking an effect of vD on LL-37 expression. However, no inverse relationship with neutrophil elastase or MMP-9 was found to support this hypothesis. Children with CF are at risk of low vD levels even if they are clinically pancreatic sufficient or if vitamin supplements are being prescribed. $\mathrm{vD}$ deficiency is associated with low levels of antimicrobial defence molecules within the airway. Whether this is a clinically important phenomenon leading to susceptibility to infection and increased inflammation will be the focus of future work.

\section{P72 FEASIBILITY OF RECRUITING NEWBORN BABIES WITH CYSTIC FIBROSIS DIAGNOSED BY NEWBORN SCREENING TO A CLINICAL STUDY WITH INVASIVE OUTCOME MEASURES}

doi:10.1136/thx.2010.150979.23

${ }^{1} \mathrm{~J}$ Chudleigh, ${ }^{1} \mathrm{~A}-\mathrm{F}$ Hoo, ${ }^{1} \mathrm{~S}$ A Prasad, ${ }^{2} \mathrm{D}$ Ahmed, ${ }^{3} \mathrm{~A}$ Bush, ${ }^{1} \mathrm{C}$ Wallis, ${ }^{2} \mathrm{~J}$ Stocks. ${ }^{1}$ Great Ormond Street Hospital for Children NHS Trust, London, UK; ${ }^{2}$ UCL Institute of Child Health, London, UK; ${ }^{3}$ Royal Brompton Hospital, London, UK

Background Newborn screening (NBS) for cystic fibrosis (CF) has been available throughout the UK since 2007. Such screening is only 